\title{
Towards A Better Understanding of Workload Dynamics on Data-Intensive Clusters and Grids
}

\author{
Hui $\mathrm{Li}^{*} \quad$ Lex Wolters \\ Leiden Institute of Advanced Computer Science (LIACS), \\ Leiden University, PO Box 9512, 2333 CA, \\ Leiden, The Netherlands
}

\begin{abstract}
This paper presents a comprehensive statistical analysis of workloads collected on data-intensive clusters and Grids. The analysis is conducted at different levels, including Virtual Organization (VO) and user behavior. The aggregation procedure and scaling analysis are applied to job arrival processes, leading to the identification of several basic patterns, namely, pseudo-periodicity, long range dependence (LRD), and (multi)fractals. It is shown that statistical measures based on interarrivals are of limited usefulness and count based measures should be trusted instead when it comes to correlations. We also study workload characteristics like job run time, memory consumption, and cross correlations between these characteristics. A "bag-of-tasks" behavior is empirically proved, strongly indicating temporal locality. We argue that pseudo-periodicity, LRD, and "bag-of-tasks" behavior are important workload properties on data-intensive clusters and Grids, which are not present in traditional parallel workloads. This study has important implications on workload modeling and performance predictions in data-intensive Grid environments.
\end{abstract}

\section{Introduction}

Grid computing is rapidly evolving as the nextgeneration platform for system-level sciences and beyond. The participating clusters serve as the main horse power in a data-intensive Grid and performance evaluation studies in such an environment require better understandings on its workload dynamics. The goal of this paper is to study the statistical properties of Grid workloads at different levels, which serve as the basis for workload modeling and performance predictions.

\footnotetext{
*Corresponding author. Email address: hui.li@computer.org. Webpage: http://www.liacs.nl/home/hli. 1-4244-0910-1/07/\$20.00 (C)2007 IEEE.
}

The contribution of this work is four-folds. Firstly, to our best knowledge this is the first comprehensive workload characterization on production clusters and Grids, at different levels including VO and user behavior. Secondly, point processes are introduced to describe job arrivals and several representations are studied. It is shown that statistical measures based on interarrivals are of limited usefulness when it comes to autocorrelations and count based measures should be trusted instead. Thirdly, the aggregation procedure and scaling analysis on job count processes enable us to understand the autocorrelation structures better. Together with the cross-correlations between characteristics, we obtain an improved understanding towards workload dynamics. Last but not least, we identify several basic patterns, such as pseudoperiodicity, long range dependence, and "bag-of-tasks" behavior. These are important properties which are not present in previous studies on parallel workloads [7]. Further research on workload modeling on Grids should potentially capture these salient properties, which could have significant implications on performance evaluation studies.

The rest of this paper is organized as follows. Section 2 describes workloads in a broader perspective. Related work on parallel supercomputer workloads, network traffic, and cluster workloads are reviewed and discussed. Section 3 introduces the definition and methodology used for the analysis. Section 4 presents the empirical studies on the realworld workload data. We analyze a variety of workloads at the Grid, cluster, and supercomputer level. VO and user behavior are investigated in depth. In Section 5 the nature and origin of workload dynamics are explained and implications on modeling and predictions are discussed. Conclusions and future work are presented in Section 6.

\section{Workloads in a Broader Perspective}

The most closely related workload studies are from parallel supercomputers. On single parallel machines a large 
amount of workload data has been collected, characterized $[18,25,7]$, and modeled [6, 18, 24]. These are solid research results and they are being used extensively for performance evaluation on parallel machines [1]. However, it should be noted that previous work focus mainly on marginal and first order statistics while correlations and second order statistics receive far less attention. The reason could be that characteristics on parallel workloads are inherently weakly-autocorrelated or short range dependent (SRD). For instance, our analysis is conducted on a representative parallel workload and it is shown that the job arrivals at larger scales are indeed short range dependent. Data-intensive workloads on clusters and Grids, on the other hand, exhibit pseudo-periodic and long range dependent behavior. Therefore second order statistics and beyond are crucial in the analysis and new methodologies should be investigated for workload analysis/modeling in Grids.

Network traffic studies are reviewed here because it includes a rich collection of advanced statistic tools for analyzing and modeling self-similar, long range dependent (LRD), and (multi)fractal behavior. The self-similar nature of Ethernet traffic is discovered in [12] and consequently a set of exact self-similar models such as fractional Brownian motion and fractional Gaussian noise are proposed as traffic models $[20,26]$. Network traffic is also shown to be long range dependent, exhibiting strong temporal burstiness $[23,2]$. Both self-similar and LRD processes are most well-known examples of general scaling processes, characterized by the scaling and power law behavior [3]. Due to its inherent multi-resolution nature, wavelets is proposed as an important tool for analysis and synthesis of processes with scaling behavior [4, 27, 3]. Multifractal models and binomial cascades are proposed for those processes with rich fractal behavior beyond second-order statistics [22, 9]. These methodologies enable us to conduct scaling analysis on Grid job arrivals and identify several important patterns.

Workload characterization on clusters with marginal statistics can be found in [14, 19]. In [19] an ON-OFF Markov model is proposed for modeling job arrivals, which is essentially equivalent to a two-phase hyperexponential renewal process. The major modeling drawback using renewal processes is that the autocorrelation function (ACF) of the interarrival times vanishes for all non-zero lags so they cannot capture the temporal dependencies in time series [11]. A more sophisticated $n$-state Markov modulated Poisson process is applied for modeling job arrivals at the Grid and VO level [16], making a step towards autocorrelations and second order statistics. Nevertheless, only limited success is obtained by MMPP because of the rich behavior and patterns hidden in Grid workloads at different levels. This paper reveals those patterns of job arrivals on

Parallel Workload Archive: http://www.cs.huji.ac.il/ labs/parallel/workload/. clusters and Grids. Moreover, more workload characteristics are studied and the cross-correlations between them are further investigated.

\section{Definition and Methodology}

\subsection{Point Processes}

Job traffic can be described as a (stochastic) point process, which is defined as a mathematical construct that represents individual events as random points at times $\left\{t_{n}\right\}$. There are different representations of a point process. An interarrival time process $\left\{I_{n}\right\}$ is a real-valued random sequence with $I_{n}=t_{n}-t_{n-1}$ and it completely describes the point process. The sequence of counts, or the count process, on the other hand, is formed by dividing the time axis into equally spaced contiguous intervals of $T$ to produce a sequence of counts $\left\{C_{k}(T)\right\}$, where $C_{k}(T)=N((k+$ 1) $T)-N(k T)$ denotes the number of events in the $k$ th interval. This sequence forms a discrete-time random process of non-negative integers and it is another useful representation of a point process. A closely related measure is a normalized version of the sequence of counts, called the rate process $R_{k}(T)$, where $R_{k}(T)=C_{k}(T) / T$.

In general, forming the sequence of counts loses information because the interarrival times between events within interval $T$ are not preserved. Nevertheless, it preserves the correspondence between its discrete time axis and the absolute "real" time axis of the underlying point process. We can readily associate correlation in the process $\left\{C_{k}(T)\right\}$ with correlation in the point process. The interarrival time process, on the other hand, eliminates the direct correspondence between absolute time and the index number so it only allows rough comparisons with correlations in the point process [17]. As we will show later, measures based on interarrival times are not able to reliably reveal the fractal nature of the underlying process and count based measures should be trusted instead.

\subsection{Statistical Measures}

No single statistic is able to completely characterize a point process and each provides a different view and highlights different properties. A comprehensive analysis towards a better understanding requires many such views. The marginal properties we study includes the moments $E\left[X^{m}\right]$ (mean and variance), the probability density, and the probability distribution. The second order statistics contains the autocorrelation function (ACF) and its Fourier transform called power spectral density or power spectrum. Scatter plot, correlation coefficients, and the crosscorrelation function are used to study correlations between distinct characteristics. The wavelet-based analysis using 


\begin{tabular}{|l|l|l|l|r|r|r|}
\hline Trace & Location & Architecture & Scheduler & \#CPUs & Period & \#Jobs \\
\hline LCG1 & Grid wide & clusters & Grid Broker & $\sim 30 \mathrm{k}$ & Nov 20-30, 2005 & 188,041 \\
\hline LCG2 & Grid wide & clusters & Grid Broker & $\sim 30 \mathrm{k}$ & Dec 19-30, 2005 & 239,034 \\
\hline NIK05 & NIKHEF, NL & PC cluster & PBS/Maui & 288 & Sep - Dec, 2005 & 63,449 \\
\hline RAL05 & RAL, UK & PC cluster & PBS/Maui & 1,000 & Oct - Nov, 2005 & 332,662 \\
\hline LPC05 & LPC, FR & PC cluster & PBS/Maui & 140 & Feb - Apr, 2005 & 71,271 \\
\hline SBH01 & SDSC, US & IBM SP & LoadLeveler & 1152 & Jan - Dec, 2001 & 88,694 \\
\hline
\end{tabular}

Table 1. Summary of workload traces used in the experimental study.
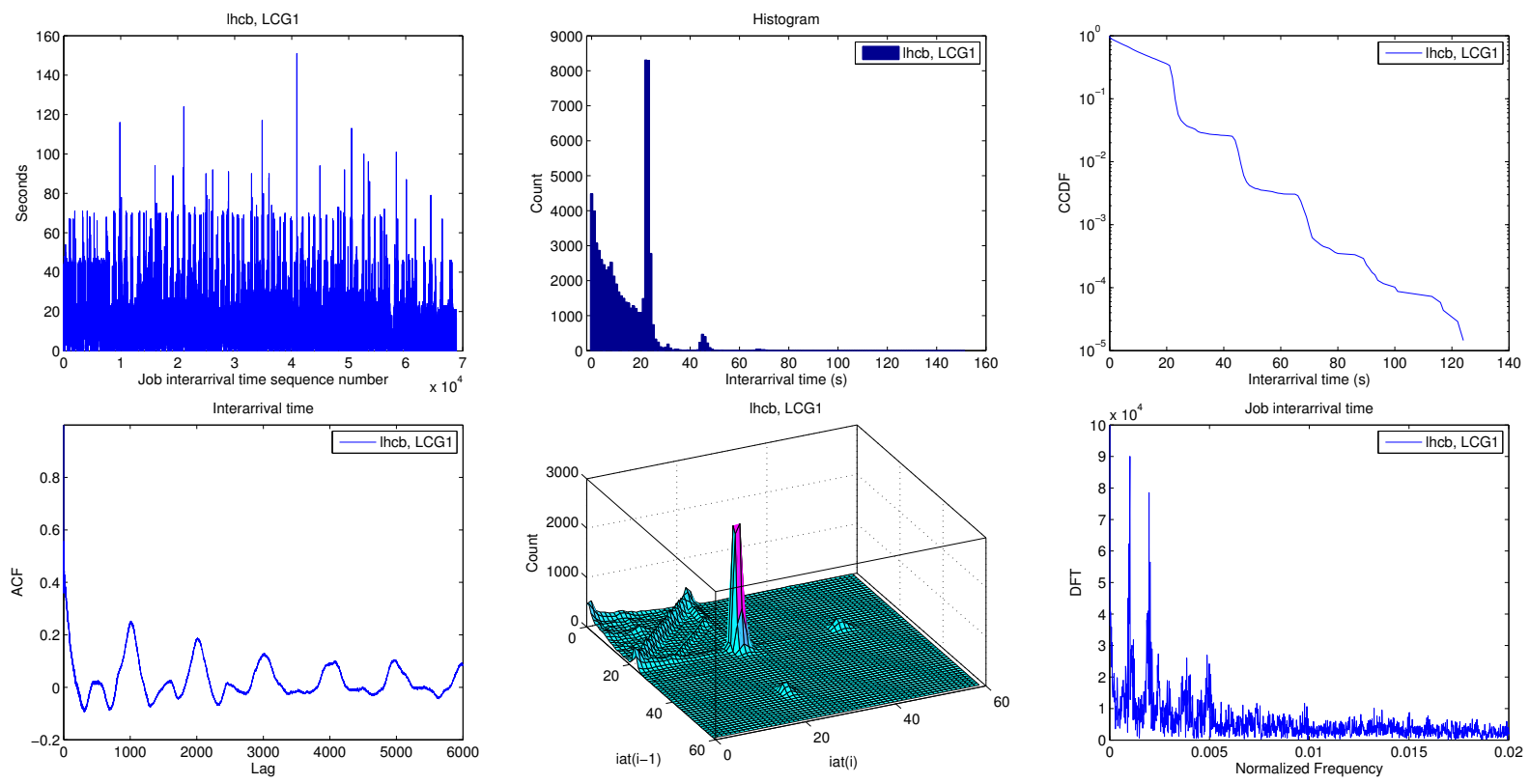

Figure 1. Plots of first and second order statistics for the interarrival time process of $l h c b, L C G 1$.

Logscale Diagram and Multiscale Diagram [3] is applied to job arrivals for identifying the scaling behavior, including fractal and long range dependence. Due to space limits we refer to [13] for a detailed discussion on various statistical measures and the scaling behavior.

\section{Application to Workload Data}

In this section we conduct experimental studies on real world workload data from production clusters and Grids. Table 1 presents a summary of workload traces under study. LCG1 and LCG2 are two traces from the LHC Computing Grid. The LCG production Grid currently has approximately 180 active sites with around 30,000 CPUs and 3 petabytes storage, which is primarily used for high energy physics (HEP) data processing. There are also jobs from biomedical sciences running on this Grid. Almost all the

LCG is a data storage and analysis infrastructure for the high energy physics community that will use the Large Hadron Collider (LHC) at CERN. http: / / lcg. web. cern. ch/LCG/. jobs are massively parallel tasks, requiring one CPU to process a certain amount of data. The workloads are obtained via the LCG Real Time Monitor for two periods: LCG1 consists of jobs of eleven consecutive days from November 20th to 30th in 2005, while LCG2 is from December 19 th to 30 th in the same year. At the cluster level we use traces from three data-intensive clusters. They are located at the HEP institutes in the Netherlands, UK, and France, respectively, and all participate in LCG. It should be noted that these clusters are involved in multiple different collaborations and have their own local user activities. Grid jobs from LCG only account for a part of the whole workloads, depending on the local policies. The trace $S B H O 1$ is from "Blue Horizon" in San Diego Supercomputer Center (SDSC) and it is included for comparison studies.

In the Grid community Virtual Organization (VO) is an

The Real Time Monitor is developed by Imperial College London and it monitors jobs from all major Resource Brokers in LCG therefore the data it collects are representative at the Grid level. http://gridportal. hep.ph.ic.ac.uk/rtm/. 

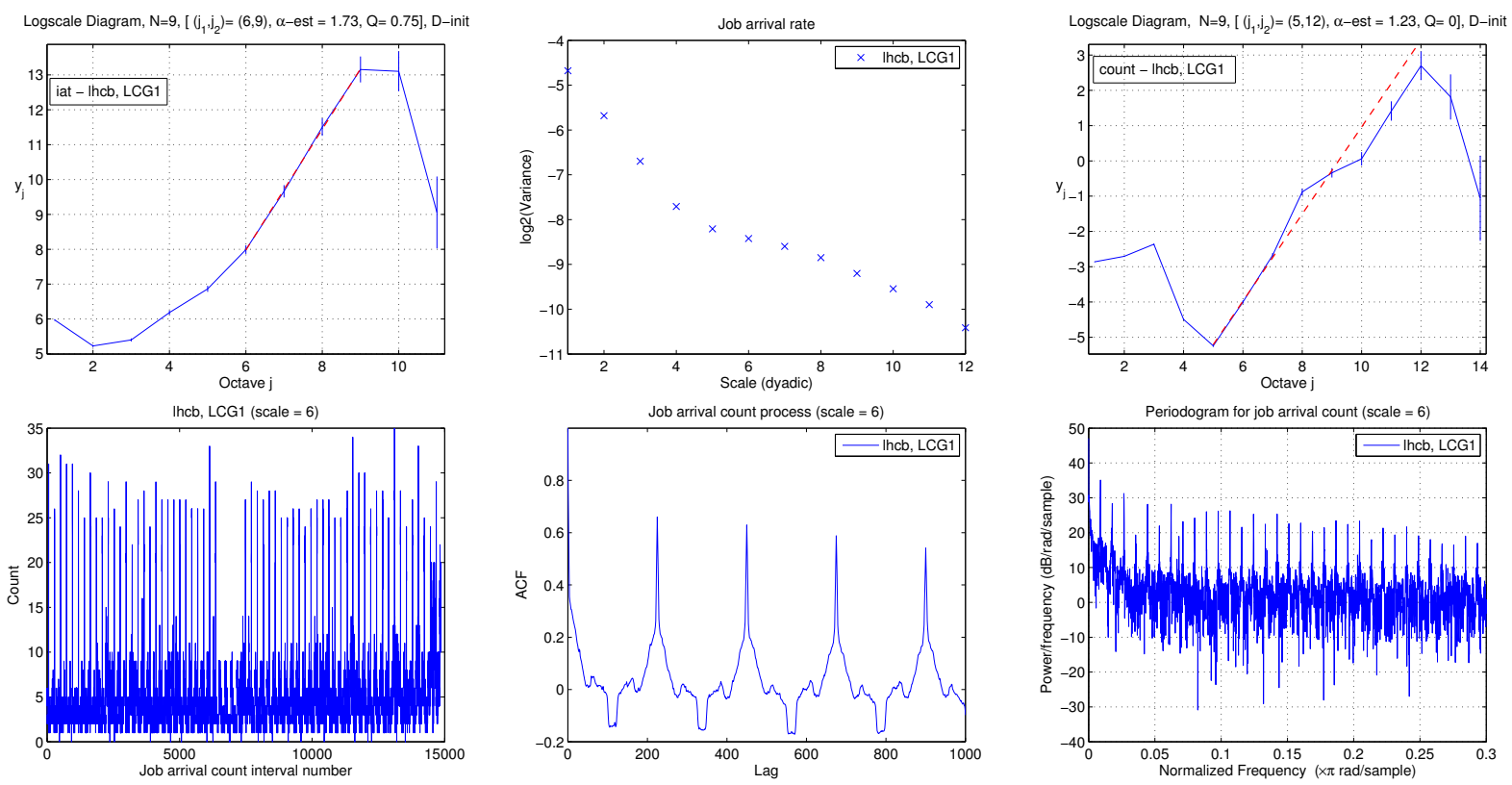

Figure 2. Plots of the scaling analysis and aggregation procedure for $l h c b, L C G 1$.

important concept [10] and one can consider a VO as a collection of entities (users, resources, etc) that belong to multiple organizations but have common goals or shared policies. In the LCG Grid VOs are mostly named after major HEP experiments, collaborations, or scientific disciplines. It is shown that the top VOs and users often dominate the workload, which means analyzing the limited number of top VOs or users could cover most of the workloads. Moreover, as we will show later, patterns emerge by simply using the nominal VO names for categorization without any sophisticated clustering treatment. From a performance evaluation perspective it is also desirable to include VO or users in the synthetic traces since most of the policy rules are based on their names. Given these motivations, our analysis mainly focuses on the VOs. Some of the VO names appeared in this paper are briefly explained here. lhcb, atlas, and $\mathrm{cms}$ are major HEP experiments in the LCG Grid. dteam is a VO mostly consisting of software monitoring and testing jobs in the Grid. hepl is a HEP collaboration between institutes in UK and US, part of which is also involved in LCG. biomed is the $\mathrm{VO}$ with biomedical applications and it contributes to $\sim 65 \%$ of $L P C 05$ jobs.

Our analysis is to apply the statistical measures discussed in Section 3.2 to each level of workloads for different characteristics. This has generated a large number of data and figures. Our interest, on the other hand, is to discover some basic pattern or patterns of the workload characteristics. Therefore the presentation of analytic results are categorized by the revealed patterns and only representative figures of each pattern are shown. In the following sections, we present the job arrival patterns first, followed by run time, memory and cross-correlations between characteristics. Interesting readers are directed to [13] for the complete results including different levels on all traces.

\subsection{Job Arrivals}

There are three basic patterns discovered for job arrivals: pseudo-periodicity, long range dependence (LRD), and (multi)fractals, which are presented subsequently in the following sections.

\subsubsection{Pseudo-Periodicity}

There are a number of VOs at the Grid and the cluster level which exhibit pseudo-periodic patterns and lhcb on LCGI is used as the example. Figure 1 shows the first and second order statistics of job interarrival times of $l h c b-L C G l$. We can see that the marginal distribution of interarrival times is not heavy-tailed, resembling exponential behavior in the y$\log$ plot. A strong deterministic component of around 20 seconds is observed in the histogram plot, which is also evidenced in the pair-correlation plot. As to the secondorder properties like the ACF and DFT certain periodicity is detected. Long-lag decaying peaks in the ACF plot correspond to the multimodals in the low frequency domain of the DFT. Nevertheless, periodicity for interarrival times does not hold for all processes belonging to this pattern. This is in accordance with the fact that interarrival based measures eliminate the direct relation with the real time axis so count based measures should be examined. 

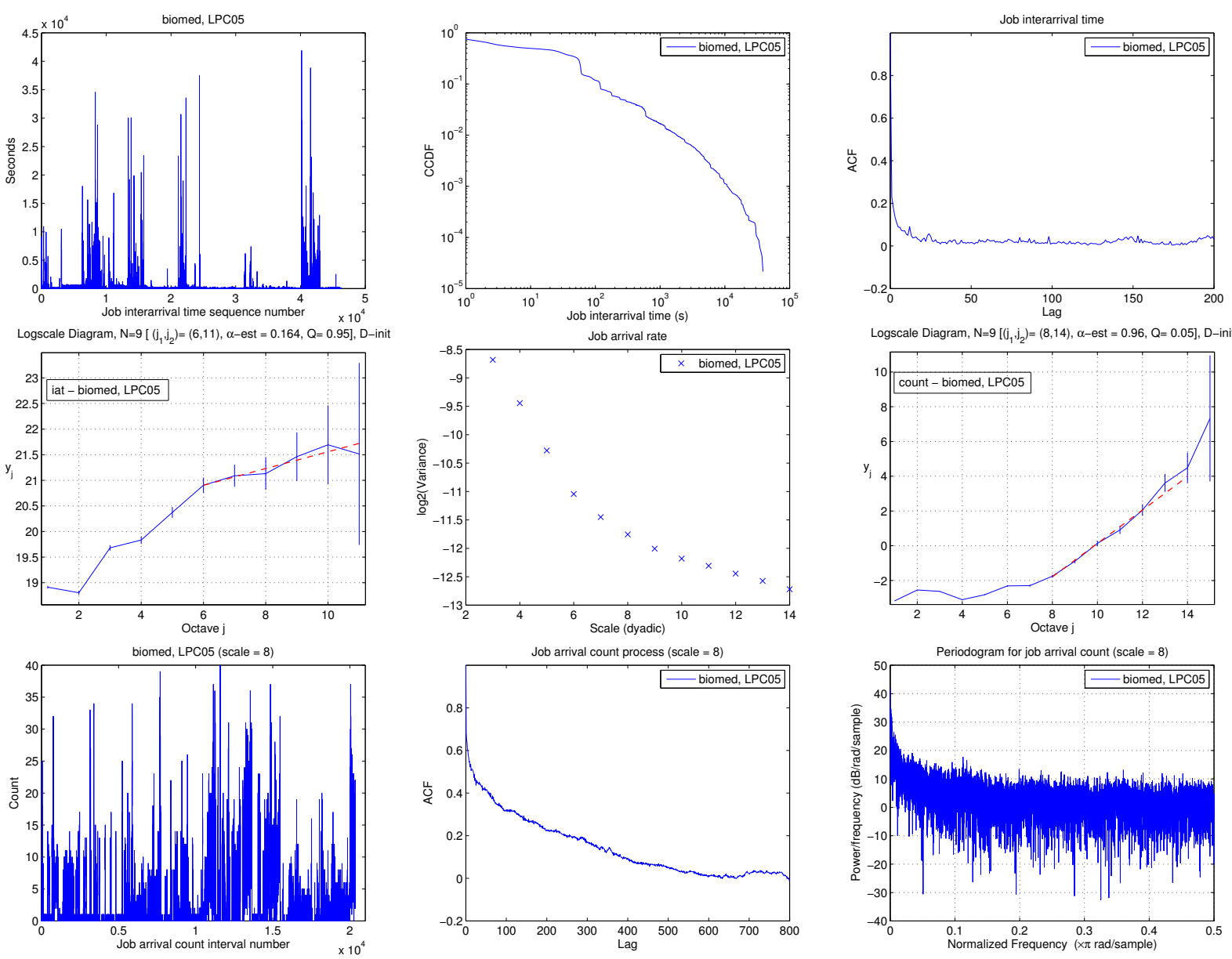

Figure 3. Plots of first and second order statistics and scaling analysis for biomed, LPCO5.

The next step naturally goes to the aggregation procedure which uses count based measures. Figure 2 plots the count process together with its ACF and power spectrum for scale $=6$. Periodicity is clearly detected by the equallyspaced peaks in the ACF plot and the multiple harmonics in the power spectrum. Stochastic fluctuations (noise) are also present in the count processes. This pattern holds for all the VOs in the same category with different base frequencies.

Scaling analysis with the aggregated variance and the Logscale Diagram are shown in Figure 2. In the variance plot the slope from scale 6 to 12 is estimated to be $\beta \approx-0.3$ $(H \approx 0.85)$. This corresponds to the Logscale Diagram with octave $j$ from 5 to 11 , showing a scaling alignment with the estimated exponent $\alpha=1.23>1$. However, the scaling analysis in this case proves to be of limited usefulness because of the strong deterministic nature of the underlying point process.

A dyadic scale is used so scale $j$ means $T=2^{j}$ seconds in the count process. This applies to all the scales in the count based measures used throughout this paper.

\subsubsection{Long Range Dependence (LRD)}

biomed-LPCO5 is used as a representative example for illustrating long range dependence. As is shown in Figure 3, the interarrival time distribution is heavy-tailed and amplitude burstiness is observed. The ACF of interarrival times, on the other hand, is short range dependent. This is in accordance with the scaling exponent estimate $\alpha=0.164$ in the Logscale Diagram in Figure 3. In the count based Logscale Diagram the scaling region is from the octave 8 (corresponding to scale 10 in the variance plot) up to the largest scale with an estimated scaling exponent $\alpha=0.96$. This type of scaling strongly suggests long range dependence behavior [3]. Plotting the count processes from several scales and their second order statistics further confirm LRD. We can see from Figure 3 that the ACF and the spectrum of scale 8 decay very slowly. It should be noticed that the scaling and LRD behavior has a certain lower bound beyond which scaling is not obeyed.

Data from real production systems is highly complex and different patterns can be observed within one process. Long 

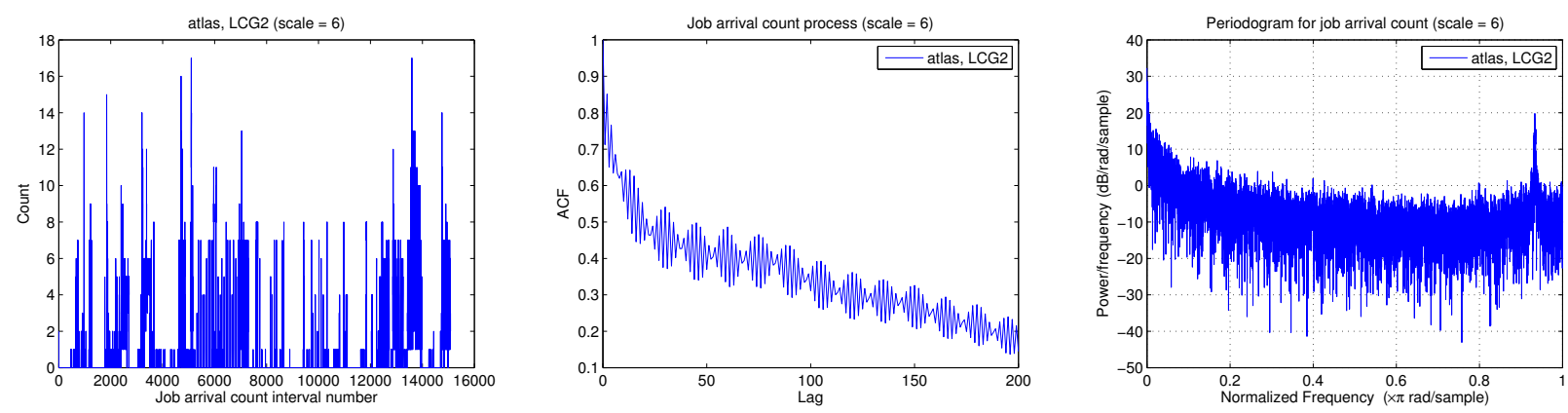

Figure 4. Plots of the first and second order statistics for atlas, LCG2.
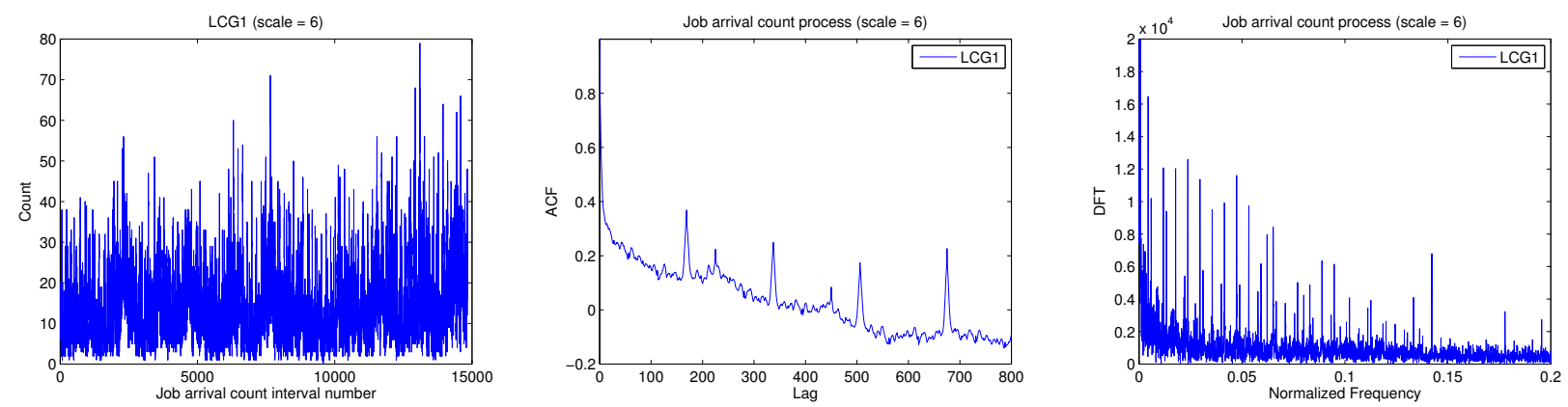

Figure 5. Plots of the first and second order statistics for $L C G 1$.

range dependence, for instance, can be mixed with periodic components. There are two types of periodic components added to a LRD process. The first type is LRD plus highfrequency periodic components and the results for atlas$L C G 2$ are plotted in Figure 4. In the pair-correlation plot a high peak around $(10,10)-$ seconds is clearly observed for job interarrival times. The ACF and the Logscale Diagram suggest that the interarrival time process is short range dependent, though. The count process, on the other hand, show a scaling region from scale 6 and above that indicates LRD (see the variance and LD plot). The aggregated count plot for scale $=6$ shown in Figure 4 further confirm the LRD argument. There is also an interesting finding of high frequency periodic components in the power spectrum. As is shown in the scale $=6$ ACF plot those periodic fluctuations are nicely aligned with the power law decay. The high frequency components can be related to the strong deterministic peak in the pair-correlation plot.

The second type of periodic behavior contains multiple components, mostly concentrated in the lower frequency domain. This type is usually found in the aggregated whole trace with mixed deterministic and stochastic components. The Grid level $L C G 1$ and $L C G 2$ are examples of this pattern and $L C G 1$ count plots are shown in Figure 5. The count process (scale $=6$ ) is LRD along with multiple low frequency peaks. We can even relate these peaks with the major VOs. Remember that we discussed the periodic patterns to which lhc $b$ belong. If we cross-refer the ACF plots we can find that the 240-minute peak is contributed by lhcb. This indicates that the count/rate processes are formed by aggregations of VO processes. For larger scales periodic patterns become dominant mostly due to hourly or daily cycles.

\subsubsection{Fractals}

Figure 6 show hepl on RALO5 as an example for (multi)fractals. The interarrival time process is short range dependent. The Logscale Diagram of the count process exhibits biscaling [3]. The scaling concentrated at the lower scales indicates the fractal nature of the sample path. The alignment at higher scales, on the other hand, resembles that of a stationary SRD process. This is further visualized in Figure 6 for scale $=6$ with quickly vanishing ACF lags and a white-noise like spectrum. For testing multifractality we plot the Multiscale Diagram of the count process ("blue circle", middle-right in Figure 6). A simulated fractional Gaussian noise (fGn) with $H=0.8$ is also shown as a monofractal reference ("red cross"). On the left we can see that the $\zeta_{q}$ of fGn (star-dotted line) is linear to $q$ while the hep1-RAL05 count process (circle-dashed line) is nonliear, indicating multifractal scaling. This corresponds to the plot on the right: the $h_{q}$ of the count process departs heavily from the horizontal line-like fGn. A multifractal model is needed to capture the scaling behavior of such patterns [22]. 

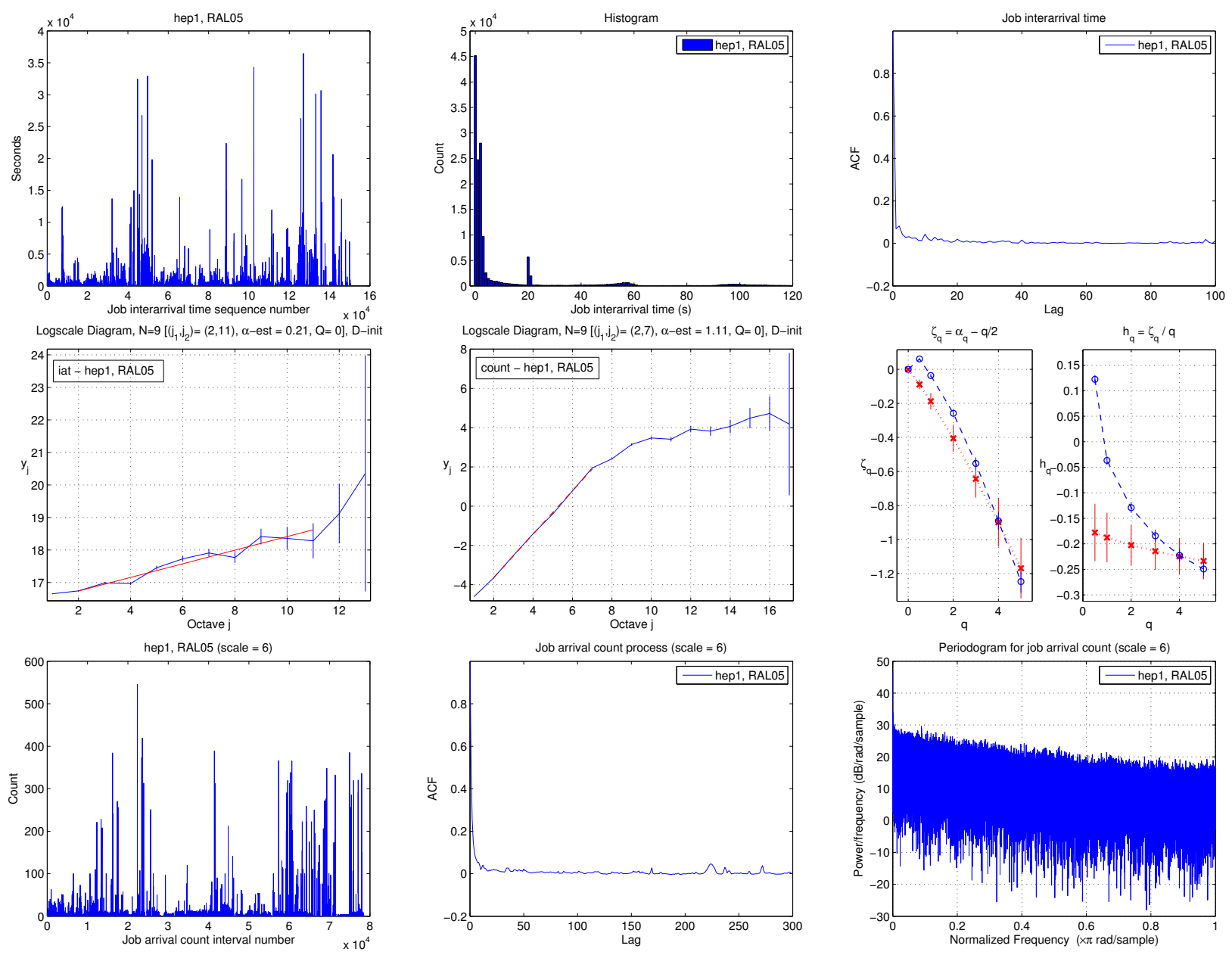

Figure 6. Plots of first and second order statistics and scaling analysis for hep1, RALO5.

\subsection{Job Run time, Memory, and Cross Correlations}

In this section we turn our focus into characteristics such as run time and memory. On one hand, first order statistics is plotted to see how data values are distributed. On the other hand, autocorrelations are used to examine second order properties in the sequence of data when ordered ascendantly by the arrival times. Figure 7 plots the marginal distributions and autocorrelations for job run time and memory of $l h c b$ on NIK05. The distributions of run times are highly multimodal with specific values, meaning that the applications within one VO are more similar to each other with clustered values of running times. Similar results are observed for memory consumption. Physically we can interpret that these jobs belong to multiple applications within a certain large scale scientific experiment or discipline. Run times and memories with similar values also turn to appear subsequently in time, which is evidenced by the fluctuating horizontal lineups in the sequence plot. It is not surpris- ing to see the strong autocorrelations in the sequences of run times or memories. One explanation of these observations is that the computing environment at the cluster level is more homogeneous compared to the Grid so less variations are expected on job run times and memories. The nature of data-intensive applications (bags of tasks) and similarity resulted by VO categorization should lead to an even stronger degree of temporal locality [8].

It is also interesting to see how the interarrival times are jointly distributed with the sequences of similar values. This helps to correlate arrivals and run times/memories and identify the "bag-of-tasks" phenomenon on data-intensive environments. Figure 7 shows the scatter plots of run times and memories against interarrival times for $l h c b-N I K 05$. We can see that job run times and memories are heavily clustered in the ranges of small interarrival times. This is to say that not only similar values appear in a sequence, but also times between arrivals in a sequence are relatively small, indicating the "bag-of-tasks" behavior. Figure 7 also contains a scatter 

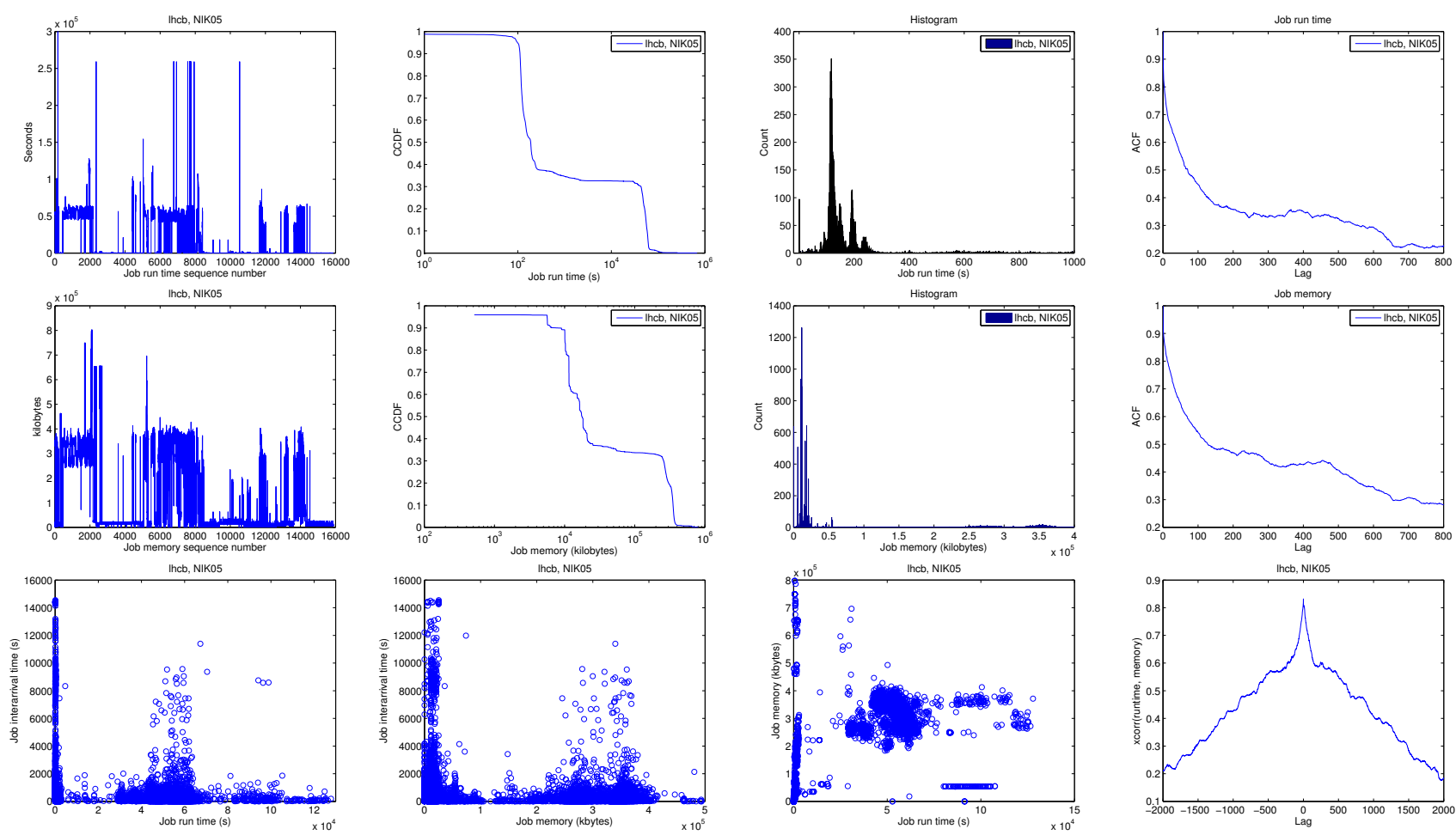

Figure 7. Plots of the first and second order statistics for run time and memory as well as crosscorrelations between them for lhcb, NIK05.

plot of run time against memory and its cross-correlation function for lhcb-NIK05. Correlation coefficients calculated by Pearson's as well as by Spearman' rank are given in Table 2. Among the three VOs lhcb-NIK05 shows the strongest correlation between run time and memory. We can see it from the clustering in the scatter plot and the slow-decaying cross-correlation function, confirmed by a high correlation coefficient. For the other two VOs weak to moderate correlation coefficients are obtained, however, correlation coefficients are used only in combination with other measures due to their inherent limitations (especially Pearson's). Referring to the complete results [13] we can conclude that temporal locality and "bag-of-tasks" behavior are clearly evidenced for VOs on data-intensive clusters. At the Grid level this behavior is not clearly observable for all the VOs due to heterogeneity, but it is present in the workloads. For the parallel supercomputer SBHO1 no bags of tasks can be identified, even for uses whose marginal distribution of run times shows strong multimodality.

\section{Discussions}

In this section we discuss the nature and origin of the discovered patterns. One of the primary purposes for statistical studies is to inspire and assist workload modeling and per-

\begin{tabular}{|l|c|c|}
\hline Trace & Pearson's & Spearman's Rank \\
\hline biomed-LPC05 & 0.173 & 0.695 \\
\hline lhcb-NIK05 & 0.756 & 0.826 \\
\hline hep1-RAL05 & 0.013 & 0.456 \\
\hline
\end{tabular}

Table 2. Correlation coefficients (CC) for run time against memory of VOs on clusters.

formance predictions. We will cover these topics in greater detail as well.

\subsection{The Nature of Workload Dynamics}

Our focus in this paper is on data-intensive computing environments whose workloads consist of flows of massively-parallel single-CPU tasks. By looking at the current workload structure of production clusters and Grids, together with the booming factor of computing-oriented solutions to system-level sciences such as bioinformatics, we can envision that data-intensive applications will contribute to a majority of workloads running on current and future Grids. This type of applications is also well suited to run on a heterogeneous Grid environment because of its loosely- 
coupled and data-parallel nature. Truly parallel applications like those on traditional parallel supercomputers, on the other hand, are more tightly-coupled with inter-process communications which make them more difficult moving from one architecture to another. Based on the different nature of applications and domain properties, it is expected that data-intensive workloads possess structures and patterns which are departed from those based on parallel supercomputers. We start our quest on the origin of job arrival dynamics.

There are three patterns that we discovered for dataintensive job arrivals. The first one exhibits strong periodicity, which suggests certain deterministic job submission mechanisms. lhcb is a large HEP experiment in the LCG Grid with the largest portion of production jobs. If we take into account that close to $90 \%$ of $l h c b$ jobs (around 60,000) are from a single "user" during the eleven consecutive days in $L C G 1$, we can assume that scripts are used to submit those jobs, which are deterministic in nature. It can also be interpreted that automated tasks need to be implemented to process such a huge amount of scientific data. Periodicity can also be originated from testing and monitoring jobs in the Grid such as those from dteam. dteam stands for "deployment team" and it is dedicated for a continuously functioning and operating Grid. Mostly testing and monitoring jobs are initiated automatically by software in a periodic fashion. The periodic pattern is also observed for VOs at the cluster level. We consider it as one basic pattern that originates from automated submission schemes, which is shown to be present in large-scale data processing environments. The second pattern is long range dependent (LRD) and it applies to many production VOs. This can be partially explained by the repetitive executions of several main applications from major users in the VO. A typical user would submit sequences of tasks with a heavy-tailed inter-submission time. This behavior shows temporal burstiness, which is argued in [5] that it essentially originates from a priority selection mechanism between tasks and non-tasks waiting for execution. LRD forms the second basic pattern that characterizes data-intensive job arrivals. If we combine periodicity and LRD, some interesting patterns emerge. The process can be long range dependent with high frequency oscillations, rooting from the short-period repetitions of job arrival rates at small time scales. The process can also be LRD with multiple lower frequency components, which is mainly due to the addictive nature of aggregation at the Grid level. There are limited data-intensive traces that show rich fractal behavior at small scales and are short range dependent at large scales. Most of the parallel supercomputer traces belong to this category.

When we take more characteristics like run time and memory into account, a "bags-of-tasks" behavior is evident for data-intensive jobs. The marginal distributions for run time and memory are highly multimodal at the VO level on clusters. Certain values not only occur subsequently, but also turn to appear within one burst period. This is again due to the nature of data-intensive applications. On parallel supercomputers, on the other hand, no such behavior is observed in the workloads.

\subsection{Modeling and Predictions}

Effective scheduling algorithm design both at the Grid and the cluster level requires experimental studies based on simulations, in which representative workload models play a crucial role. There are many modeling work being done for parallel workloads [1], but on Grids it is still emerging [16]. This paper is particularly useful for the modeling research because a set of basic patterns are identified for job arrivals on clusters and Grids, which points out directions for selecting models to fit distinctive patterns. Moreover, cross correlations between job characteristics are investigated and illustrated. As we have shown these patterns and correlation structures on Grids are very different from those which have been studied and modeled on parallel supercomputers. There is a strong need to build models that are able to capture the dynamics both within and between job characteristics for data-intensive workloads.

Another important perspective on workload analysis is how to help the research on performance predictions. Predictions of performance metrics, such as application run times and queue wait times on clusters, serve as important information for scheduling decision making at the Grid level. The main patterns that we identify for data-intensive clusters, namely periodicity, long range dependence, and temporal locality, suggest that prediction techniques based on historical data modeling would most likely work on real production systems [21, 15]. The Grid-level scheduling strategies can also take advantages of specific VO job arrival patterns. For instance, if periodicity is detected for a large production VO, scheduling heuristics can be optimized specifically for this strong deterministic behavior.

\section{Conclusions and Future Work}

In this paper we study comprehensively the first and second order statistics of workloads on clusters and Grids at different levels, including VO and user behavior. We identify several basic patterns for job arrivals and these patterns provide important clues from a modeling perspective. The sequences of job run times or memory on clusters are strongly autocorrelated and their cross-correlations are also significant. Moreover, sequences of similar values turn to appear in a burst with small interarrival times, leading to the observation of "bag-of-tasks" behavior. To sum up, pseudoperiodicity, long range dependent, and temporal locality 
("bag-of-tasks") are important characteristic properties of workloads on data-intensive clusters and Grids, which is not present in the traditional parallel workloads.

Future work naturally extends to workload modeling that tries to capture the correlation structures and patterns obtained in this paper. Experimental performance evaluation studies using simulations are needed to investigate their impact and how to improve scheduling under such workload patterns.

\section{Acknowledgments}

The LCG Grid traces are provided by the HEP e-Science group at Imperial College London. NIKO5 and RAL05 traces are provided by colleagues at NIKHEF (NL) and RAL (UK), respectively. $L P C O 5$ and SBHOI traces are obtained from Parallel Workload Archive. We want to express our gratitude to all who graciously provide us with the data. We also want to thank Michael Muskulus, Steven Lowen (Harvard), and Rudolf Riedi (Rice) for their suggestions and discussions on point processes and scaling analysis issues.

\section{References}

[1] Selected and revised papers: Workshop on job scheduling strategies for parallel processing. Lecture Notes in Computer Science. Springer, 1995 - 2005.

[2] P. Abry, R. Baraniuk, P. Flandrin, R. Riedi, and D. Veitch. The multiscale nature of network traffic: discovery, analysis, and modelling. IEEE Signal Processing magazine, 19(3):28-46, May 2002.

[3] P. Abry, M. S. Taqqu, P. Flandrin, and D. Veitch. Self-Similar Network Traffic and Performance Evaluation, K. Park and W. Willinger, editors, chapter Wavelets for the analysis, estimation, and synthesis of scaling data. Wiley, 2000.

[4] P. Abry and D. Veitch. Wavelet analysis of long-range dependent traffic. IEEE Trans. on Info. Theory, 44(1):2-15, January 1998.

[5] A.-L. Barabasi. The origin of bursts and heavy tails in human dynamics. Nature, 435:207-211, 2005.

[6] W. Cirne and F. Berman. A comprehensive model of the supercomputer workload. In proceedings of IEEE 4th Annual Workshop on Workload Characterization, 2001.

[7] D. G. Feitelson. Workload modeling for performance evaluation. LNCS, 2459:114-141, 2002.

[8] D. G. Feitelson. Workload Modeling for Computer Systems Performance Evaluation. draft version 0.7, 2006.

[9] A. Feldmann, A. C. Gilbert, and W. Willinger. Data networks as cascades: Investigating the multifractal nature of internet WAN traffic. In SIGCOMM, pages 42-55, 1998.

[10] I. Foster, C. Kesselman, and S. Tuecke. The anatomy of the Grid: Enabling scalable virtual organizations. Lecture Notes in Computer Science, 2150, 2001.

[11] D. L. Jagerman, B. Melamed, and W. Willinger. Stochastic modeling of traffic processes. Frontiers in Queueing: Models, Methods and Problems, 1996.
[12] W. Leland, M. Taqqu, W. Willinger, and D. Wilson. On the self-similar nature of ethernet traffic (extended version). IEEE/ACM Trans. on Networking, 2(1):1-15, 1994.

[13] H. Li. Complete data and results for workload dynamics on clusters and grids. Technical Report 2006-04, Leiden Institute of Advanced Computer Science, Leiden University, 2006.

[14] H. Li, D. Groep, and L. Wolters. Workload characteristics of a multi-cluster supercomputer. LNCS, 3277:176-193, 2005.

[15] H. Li, D. Groep, and L. Wolters. Mining performance data for metascheduling decision support in the grid. Future Generation Computer Systems, 23:92-99, 2007.

[16] H. Li and M. Muskulus. Analysis and modeling of job arrivals in a production grid. ACM SIGMETRICS Performance Evaluation Review, March 2007.

[17] S. B. Lowen and M. C. Teich. Fractal-Based Point Processes. John Wiley and Sons, Inc., 2005.

[18] U. Lublin and D. G. Feitelson. The workload on parallel supercomputers: modeling the characteristics of rigid jobs. J. Para. and Dist. Comput., 63(11):1105-1122, 2003.

[19] E. Medernach. Workload analysis of a cluster in a grid environment. In proceedings of 11th workshop on Job Scheduling Strategies for Parallel processing, 2005.

[20] V. Paxson. Fast, approximate synthesis of fractional gaussian noise for generating self-similar network traffic. Computer Communications Review, 27(5):5-18, Oct. 1997.

[21] Y. Qiao, J. Skicewicz, and P. Dinda. Multiscale predictability of network traffic. Technical Report NWU-CS-02-13, Department of Computer Science, Northwestern University, 2002.

[22] R. H. Riedi, M. S. Crouse, V. J. Ribeiro, and R. G. Baraniuk. A multifractal wavelet model with application to network traffic. IEEE Transactions on Information Theory, 45(3):992-1019, April 1999.

[23] R. H. Riedi and W. Willinger. Self-Similar Network Traffic and Performance Evaluation, K. Park and W. Willinger, editors, chapter Toward an Improved Understanding of Network Traffic Dynamics. Wiley, 2000.

[24] B. Song, C. Ernemann, and R. Yahyapour. Parallel computer workload modeling with markov chains. LNCS, 3277:4762, 2004.

[25] M. S. Squillante, D. D. Yao, and L. Zhang. The impact of job arrival patterns on parallel scheduling. ACM SIGMETRICS Performance Evaluation Review, 26(4):52-59, Dec. 1999.

[26] J. L. Vehel and R. Riedi. Fractional brownian motion and data traffic modeling: The other end of the spectrum. Fractals in Engineering, pages 185-202, 1997.

[27] D. Veitch and P. Abry. A wavelet based joint estimator of the parameters of long-range dependence. IEEE Transactions on Information Theory special issue on "Multiscale Statistical Signal Analysis and its Applications”, 45(3):878-897, April 1999. 